\title{
Parameterized Image Filtering Using fuzzy Logic
}

\author{
Shresth Gupta \\ Shri Shankaracharya College of \\ Engineering and Technology \\ Bhilai, India
}

\author{
Ekant sharma \\ Shri Shankaracharya College of \\ Engineering and Technology \\ Bhilai, India
}

\begin{abstract}
The principal source of blur in digital images arise during image acquisition (digitization) or transmission. The performance of imaging sensors is affected by a variety of factors, such as the environmental conditions during image acquisition. Blurry images are the result of movement of the camera during shooting (not holding it still) or the camera not being capable of choosing a fast enough shutter speed to freeze the action under the light conditions. For instance, in acquiring images with a camera, light levels and sensor temperature are major factors affecting the amount of blur in the resulting image.

Blur was implemented by first creating a PSF filter in MatLab that would approximate linear motion blur. This PSF was then convolved with the original image to produce the blurred image. Convolution is a mathematical process by which a signal, in this case the image, is acted on by a system, the filter, in order to find the resulting signal. The amount of blur added to the original image depended on two parameters of the PSF: length of blur (in pixels), and the angle of the blur. This thesis work is going to provide a new, faster, and more efficient noise reduction method for images corrupted with motion blur. This new filter has two separated steps or phases: the detection phase and the filtering phase. The detection phase uses fuzzy rules to determine whether a image is blurred or not. When blurry image is detected, Then we use fuzzy filtering technique focuses only on the on the real blurred pixels.
\end{abstract}

Keywords: Impulse noise, iterative filtering algorithm, Gaussian noise, edge based comparison

\section{INTRODUCTION}

The process of receiving and analyzing visual information by the human species is referred to as sight, perception or understanding. Similarly, the process of receiving and analyzing visual information by digital computer is called digital image processing [5].One of the first applications of digital images was in the newspaper industry, when pictures were first sent by submarine cable between London and New York. The term digital image processing refers to processing of a two dimensional picture by a digital computer. In other words, it implies digital processing of any two dimensional data. A digital image is an array of real or complex numbers represented by a finite number of bits. An image given in the form of a transparency, slide, photograph, and chart is first digitized and stored as a matrix of binary digits in computer memory. The digitized image can then be processed on a high resolution television monitor. For display, the image is stored in a rapid access buffer memory which refreshes the monitor at 30 frames per second to produce a visibly continuous display.

The principal source of noise in digital images arise during image acquisition (digitization) or transmission. The performance of imaging sensors is affected by a variety of factors, such as the environmental conditions during image acquisition, and by the quality of the sensing elements themselves. For instance, in acquiring images with a camera, light levels and sensor temperature are major factors affecting the amount of noise in the resulting image. Images are also corrupted during transmission principally due to interference in the channel used for transmission. For example, an image transmitted using a wireless network might be corrupted as a result of lighting or other atmospheric disturbance. There are various types of noise can be added in image.

\section{DETECTION AND FILTERING ANALYSIS}

Noise is usually quantified by the percentage of pixels which are corrupted. Corrupted pixels are either set to the maximum value or have single bits flipped over. In some cases, single pixels are set alternatively to zero or to the maximum value. This is the most common form of impulse noise and is called salt and pepper noise.

This new filter has two separated steps or phases: the detection phase and the filtering phase. The detection phase uses fuzzy rules to determine whether a pixel is corrupted with impulse noise or not. When impulse noise is detected, some parameters will be determined which will be passed to the filtering phase. After this detection, the fuzzy filtering technique focuses only on the on the real noisy pixels A digital image $a[m, n]$ described in a $2 \mathrm{D}$ discrete space is derived from an analog image $a(x, y)$ in a $2 \mathrm{D}$ continuous space through a sampling process that is frequently referred to as digitization. The $2 \mathrm{D}$ continuous image $a(x, y)$ is divided into $N$ rows and $M$ columns. The intersection of a row and a column is termed a pixel. The value assigned to the integer coordinates $[m, n]$ with $\{\mathrm{m}=0,1,2, \ldots, \mathrm{M}-1\}$ and $\{\mathrm{n}=0,1,2, \ldots, \mathrm{N}-1\}$ is $a[m, n]$. In fact, in most cases $a(x, y)$ which we might consider to be the physical signal that impinges on the face of a $2 \mathrm{D}$ sensor is actually a function of many variables including depth (z), 
color $(\lambda)$, and time $(t)$. Unless otherwise stated, we will consider the case of 2D, monochromatic, static images.

Local operations produce an output pixel value $b[\mathrm{~m}=\mathrm{mo}$, $\mathrm{n}=\mathrm{no}$ ] based upon the pixel values in the neighborhood of $\mathrm{a}[\mathrm{m}=\mathrm{mo}, \mathrm{n}=\mathrm{no}]$. Some of the most common neighborhoods are the 4-connected neighborhood and the 8-connected neighborhood in the case of rectangular sampling and the 6-connected neighborhood in the case of hexagonal sampling illustrated in figure.

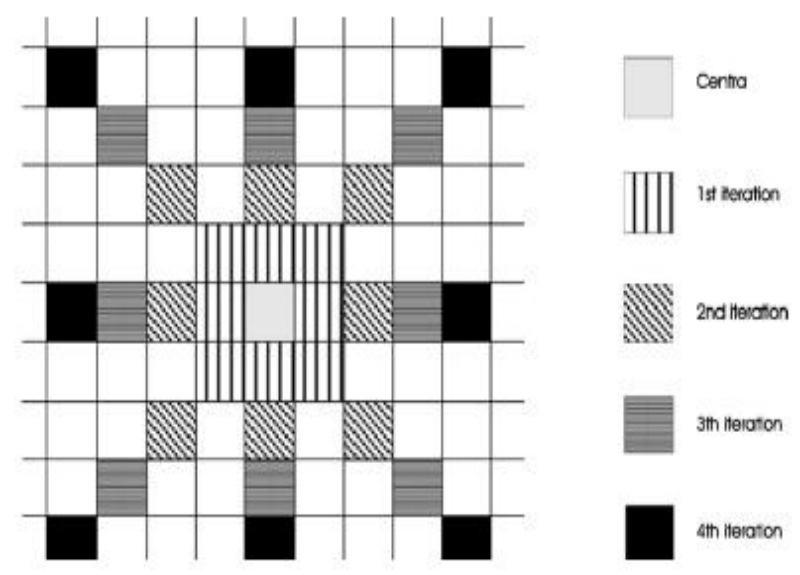

Fig. 1. Hexagonal sampling in 6-connected neighborhood

In Image representation one is concerned with the characterization of the quantity that each picture element represents [3]. An image could represent luminance of objects in a scene, the absorption characteristics of the body tissue, the radar cross section of the target, the temperature profile of the region or the gravitational field in an area. In general, any two dimensional function that bears information can be considered an image.

\section{TRADITIONAL TACTICS}

An adaptive approach to solve the restoration problem in which filtering is conditioned on the current state of the algorithm. The state variable is defined as the output of a classifier that acts on the differences between the current pixel value and the remaining ordered pixel values inside a window centered around the pixel of interest. This scheme is undoubtedly one of the robust and simple scheme but it fails in preserving the finer details of the image.

Z. Wang and D. Zhang. proposed Progressive-Switching Median filter. It is a median based filter, which works in two stages. In the first stage an impulse detection algorithm is used to generate a sequence of binary flag images [1].. This binary flag image predicts the location of noise in the observed image. In the second stage noise filtering is applied progressively through several iterations. This filter is a very good filter for fixed valued impulsive noise but for random values the performance is abysmal.

T. Chen and H. R. Wu. proposed Adaptive Centre Weighted Median Filter [10]. This work is an improvement of previously described Centre Weighted Median (CWM) filter. It works on the estimates based on the differences between the current pixel and the outputs of the CWM filters with varied center weights. These estimates decide the switching between the current pixel and median of the window. This is a good filter and is robust for a wide variety of images. But it is inefficient in recovering the exact values of the corrupted pixels.

X. Xu and E. L. Miller. proposed Adaptive Two-Pass Median filter. As the name suggests it employs median filter on the noisy image twice. This adaptive system tries to correct for false replacements generated by the first round of median filtering operation. Based on the estimated distribution of the noise, some pixels changed by first median filter are replaced by their original values and kept unchanged in the second median filtering. And in the second round it filters out the remaining impulses. Even though the filter gives some good results in terms of noise suppression but spoiling of good pixels is more and it results in overall poor performance.

\section{ENHANCEMENT WITH FUZZY STRATEGY}

Fuzzy logic was first introduced in the 1965 as a new way to represent vagueness in everyday life. The definition of fuzzy logic as a superset of conventional(Boolean) logic that has been extended to handle the concept of partial truth values between "completely true" and "completely false". By this definition, fuzzy logic departs from classical two-valued set logic. It uses soft linguistic system variables and a continuous range of true values in the interval $[0,1]$, rather than strict binary values. It is basically a multi valued logic that allows intermediate values to be defined between conventional evaluations like yes/no or true/false, etc. Notions like rather warm or pretty cold can be formulated mathematically and processed by computers."

Fuzzy logic is also a structured, model-free estimator that approximates a function through linguistic input/output associations. Fuzzy logic is a powerful, yet straight forward, problem solving technique with wide spread applicability, especially in the areas of control and decision making [6]. Fuzzy Logic was first invented as a 
representation scheme and calculus for uncertain or vague notions. It allows more human-like interpretation and reasoning in machines by resolving intermediate categories between notations such as true/false, hot/cold etc used in Boolean logic In this context, Fuzzy Logic is a problemsolving control system methodology that lends itself to implementation in systems ranging from simple, small, embedded micro-controllers to large, networked, multichannel PC or workstation-based data acquisition and

control systems. It can be implemented in hardware, software, or a combination of both. FL provides a simple way to arrive at a definite conclusion based upon vague, ambiguous, imprecise, noisy, or missing input information. FL's approach to control problems mimics how a person would make decisions, only much faster

\section{FUZZY FILTERING OF BLURRED IMAGES}

After the detection of blurred images from above traditional tactics the filtering is done. For this there is need to calculate some parameters which are used to construct the fuzzy set more or less impulse noise [6]. Afterward the iteration process is done for the filtering phase based on the membership functions that represents the fuzzy set. The obtained membership functions is a simplification of the obtained noise histogram.

In this if the membership function value greater then threshold value. If the membership value greater than threshold value then image is blurred. The extreme large value cause wide membership function that results some kind of blurring of the image.

The filtering step of first iteration is given in the algorithm .

This method is based on the membership function. The

pixels which are the part of the support of the fuzzy set are blurred have to be filtered, otherwise leave pixels unchanged. A $3 \times 3$ window around the filtered pixel is used. If the output image is the same as the input image(A) then the filter method is called recursive; otherwise non-recursive.

\section{A. Algorithm for the first iteration \\ input : A : The noisy image with impulse noise.}

$\square(A(i, j))$ : the membership degree for the fuzzy set more or less impulse noise.
F: the output image.

Steps:

(1) FOR each border pixel $(i, j) \square A$

(2) Perform comparison with neighbor pixel in window of $3 \times 3$

(3) if the blur detected in image, perform the fuzzy filtration

(4) Replace the pixel in window by the fuzzy derived pixels

(5) If there is no blur detected by neighbor comparison

i. Perform the edge based comparison within window

ii. If it satisfy then perform fuzzy filtration

iii. Replace the pixel by new fuzzy derived pixels

\section{(6) END FOR}

After the first iteration, it is possible as a side effect (especially with high initial blurred pixel) that the there is a number of moving pixel. To reduce these noisy pixels, some more (recursive) iterations are provided that are quite similar to the first one. In each iteration, we use the modified image of the previous performed iteration and a different window as shown in Fig. 5.6 around a given pixel. Fig.5.6 shows the neighborhood windows used in the first, second, third, and fourth iteration. The changing window is used to avoid future clustering and also speeds up the execution time. In addition to the different window the modification of the membership function "more or less impulse noise" (for the mth iteration ) is also done by changing the parameters. This change is going to reduce the slope of the membership functions, and therefore the amount of investigated pixels for an image A, It will speed up the execution time cause the amount of noisy pixels was already reduced in the previous iteration.

\section{B. Algorithms for the next filtering iterations $(m \square 2)$}

Input : F : The output image of the previous iteration

Steps:

(1) FOR each border pixel $(i, j) \square A$

(2) Perform comparison with neighbour pixel in window of $3 \times 3$

(3) If the blur detected in image, perform the fuzzy filtration

(4) Replace the pixel in window by the fuzzy derived pixels 

comparison

i. Perform the edge based comparison within window

ii. If it satisfy then perform fuzzy filtration

iii. Replace the pixel by new fuzzy derived pixels

\section{(6) END FOR}

To stop the iteration procedure there should be some criteria. During the first iteration every pixel is checked one by one. If the pixel value does not depend belong to the support of the fuzzy set more or less impulse noise then this pixel value is not going to changed, not only in this iteration neither in other ones. By remembering only the positions of pixels, whose pixel value is an element of the support of the fuzzy set more or less noise, one can drastically reduce the scanning amount in the next iterations. By applying these rules the iteration may stop and the filtered result can be obtained.

\section{CONCLUSION AND FUTURE WORK}

It primarily focuses on impulsive \& Gaussian noise suppression from images. A new two step filter (FIDRM), which uses a fuzzy detection and an iterative filtering algorithm, has been presented. This filter is especially developed for reducing all kinds of impulse noise (not only salt and pepper noise). Its main feature is that it leaves the pixels which are noise-free unchanged. Experimental results show the feasibility of the new filter. A numerical measure, such as the PSNR, and visual observations show convincing results for grayscale images. But the filter does not gives good result for the Gaussian noise. Finally, this new method is easy to implement and has a very low execution time.

As it has been stated that the proposed technique is not good for Gaussian noise removal , investigation may be carried out in this direction. Development of parallel algorithms can also be done to counter attack the computational overhead.
In this method we have used fuzzy logic for noise detection. Investigation may be carried out to use neural network for detection of noisy pixels in the image and fuzzy logic to remove the detected noise from the image .

\section{REFERENCES}

[1] T. G. Stockham, Jr., "Image processing in the context of a visual model," Proc. IEEE, vol. 60, no. 7, pp. 828-842, Jul. 1972.

[2] B. Bhanu, J. Peng, T. Huang, and B. Draper, "Introduction to the special issue on learning in computer vision and pattern recognition," IEEE Trans. Syst., Man, Cybern. B, Cybern., vol. 35, no. 3, pp. 391396, Jun. 2005.

[3] C. Munteanu and A. Rosa, "Gray-scale image enhancement as an automatic process driven by evolution," IEEE Trans. Syst., Man, Cybern. B, Cybern., vol. 34, no. 2, pp. 1292-1298, Apr. 2004.

[4] M. Hanmandlu, O.P. Verma, N.K. Kumar, M. Kulkarni, A novel optimal Fuzzy system for color image enhancement using bacterial foraging, IEEE Trans. Inst. Meas. 58 (8) (2009) 2867-2879.

[5] R.C. Gonzalez, R.E. Woods, Digital Image Processing, AddisonWesley, Reading, MA, 1992.

[6] M. Russo, G. Ramponi, A fuzzy operator for the enhancement of blurred And noisy images, IEEE Trans. Image Process. 4 (August (8)) (1995) 1169-1174. 